\title{
Endocarditis of Native Valve due to Proteus mirabilis: Case Report and Literature Review
}

\author{
Beatrice Tiri ${ }^{1} \cdot$ Giulia Priante $^{2} \cdot$ Alessandro Mariottini $^{3} \cdot$ Emanuela Sensi $^{4} \cdot$ Sara Gioia ${ }^{5} \cdot$ Monya Costantini $^{6}$. \\ Paolo Andreani ${ }^{3} \cdot$ Lucia Assunta Martella $^{2} \cdot$ Carlo Vernelli $^{2} \cdot$ Stefano Cappanera ${ }^{1}$
}

Accepted: 18 December 2020 / Published online: 4 January 2021

(C) The Author(s) 2021

\begin{abstract}
Endocarditis due to Proteus mirabilis is very uncommon and the optimal surgical and/or antibiotic treatment is not well defined. Guidelines from the AHA and ESC recommend prolonged courses of combined antibiotic therapy but information regarding the clinical presentation, the choice of treatment, the surgical management, and the duration of therapy can only be taken from clinical cases reported in literature. We describe a case of native valve endocarditis due to Proteus mirabilis, successfully treated with antibiotic therapy alone with a review of the relevant literature on this topic.
\end{abstract}

Keywords Infective endocarditis $\cdot$ Proteus mirabilis $\cdot$ Antibiotic therapy

\section{Introduction}

Infective endocarditis (IE) is a life-threatening condition. If left untreated, it can have adverse consequences including elevated mortality. The usual treatment involves a prolonged course of antibiotics with up to $40-50 \%$ of patients needing valve replacement during initial hospital admission [1]. The most common organisms implicated are Staphylococci, Streptococci, and Enterococci; Gram-negative agents are rarely implicated [2]. Proteus mirabilis is one such pathogen that frequently appears in the bloodstream during urinary tract infection but rarely results in endocarditis. The best antibiotic

This article is part of the Topical Collection on Medicine

Beatrice Tiri

tiri.beatrice@gmail.com

1 Antimicrobial Stewardship Unit, Department of Medicine, "S. Maria” Hospital, via Tristano di Joannuccio 1, 05100 Terni, TR, Italy

2 Infectious Diseases Clinic, Department of Medicine, "S. Maria" Hospital, Terni, Italy

3 Hematology and Microbiology Laboratory, "S. Maria” Hospital, Terni, Italy

4 Department of Critical Care Medicine and Anesthesiology, "S. Maria" Hospital, Terni, Italy

5 Institute of Legal Medicine, University of Perugia, Perugia, Italy

6 Pharmacy Unit, "S. Maria” Hospital, Terni, Italy treatment for these patients is currently unknown; guidelines from the AHA and ESC recommend prolonged courses of combined antibiotic therapy $[1,3]$ but information regarding the clinical presentation, the choice of treatment, the surgical management, and the duration of therapy can only be taken from clinical cases reported in literature. Although a systematic review was recently published [4], our aim was to compare data, investigate clinical characteristics of patients and risk factors, type of treatment, duration of therapy with a focus on native valve endocarditis. We describe a case of native valve endocarditis due to Proteus mirabilis, successfully treated with antibiotic therapy alone and a literature review on this topic (through 1 November 2020).

\section{Case Report}

A 86-year-old female with a history of aorta arch replacement in 2015 and atrial fibrillation in medical treatment was admitted to our Hospital with fever, urinary burning, and altered mental status. At the time of admission, the patient's temperature was $39^{\circ} \mathrm{C}$, the blood pressure was $90 / 60 \mathrm{mmHg}$, and the heart rate was 94 beats per minute. No focal neurological signs were present. Cardiac examination was negative and the lungs were clear to auscultation. Laboratory investigation revealed the following measurements: white blood cell count $14.2 \times$ $10^{3}$ cells $/ \mu \mathrm{L}$ with $90 \%$ neutrophils, creatinine $2.57 \mathrm{mg} / \mathrm{dL}$ (0.40-1.10 mg/dL), and C-reactive protein (CRP) $13.22 \mathrm{mg} /$ 
dL (0.00-0.75 mg/dL). Sequential Organ Failure Assessment (SOFA) Score was 3 points. The urine sediment showed 500 white blood cells and bacteria on microscopy examination.

Renal ultrasound showed bilateral non-obstructive kidney stones with no urologic indication to extraction or dissolution. Two blood culture sets and urine sample were drawn, and an empiric therapy with ciprofloxacin iv 400 bid was started.

ESBL-negative Proteus mirabilis, resistant to ciprofloxacin and trimethoprim/sulfamethoxazole, was cultured both from blood (Table 2) and urinary culture samples. After a questionable transthoracic cardiac ultrasound, the suspicion of endocarditis persisted and the patient underwent transesophageal echocardiogram (TEE) which revealed a mobile mass measuring $12 \mathrm{~mm}$ attached to the anterior leaflet of the mitral valve and a moderate regurgitation.

The patient fulfilled the Duke clinical criteria for definite endocarditis based on the trans-esophageal echocardiographic findings and positive blood culture (two major criteria). The surgeon did not pose a clear indication for valvular replacement but a "wait and see" indication and a revaluation by TEE after 2 weeks of antibiotic therapy.

The patient was treated with intravenous ceftazidime $2 \mathrm{~g} \times$ 2/day and gentamicin $160 \mathrm{mg} /$ day according to renal function. During the first week of hospitalization, the patient reported a sudden visus reduction. The fundoscopic examination and formal ophthalmology evaluation showed a posterior pole ischemic edema of the retina to be reported to a supratemporal branch retinal artery occlusion due to embolic event. After 1 week of antibiotic therapy, surveillance blood cultured was negative and after 2 weeks, TEE showed a reduction of the vegetation. Gentamicin was stopped and ceftazidime was continued. After 4 weeks of antibiotic treatment, the patient was discharged with a home therapy ertapenem for 4 weeks. After 8 weeks, at the end of antibiotic treatment, the TEE showed an absence of vegetation. The serum creatinine value was $1.66 \mathrm{mg} / \mathrm{dL}$ and the CRP was $1.26 \mathrm{mg} / \mathrm{dL}$.

\section{Results}

We searched the literature using the Pubmed database (http:// www.ncbi.nlm.nih.gov/pubmed). Query terms were "Proteus" and "Endocarditis." To date, only 13 cases about IE due to Proteus spp. were published that include sufficient clinical details reported in Table 1. Including our clinical case, the mean age of the patients was 54.1 years (range: $25-$ 86 years). Eight $(57.1 \%)$ were female, and $6(42.9 \%)$ were male. Considering the cases that reported the presence of urinary tract infection $(9 / 14)$, in $8 / 9(88.8 \%)$ cases, the patients had a concomitant urinary tract infection due to Proteus spp. Nephrolithiasis was not reported in $8 / 14(57.1 \%)$. In the remaining 6/14, nephrolithiasis was present in 5/6 (83.3\%), in 1 case $(16.7 \%)$ was absent. In $10 / 14$ case, the vegetation was attached to a native valve $(71.4 \%)$, mitral valve in $8 / 10$ cases, and aortic valve in $2 / 10$ cases. Instead, in $4 / 14$ cases $(28.6 \%)$, the vegetation was attached to a prosthetic valve; in $2 / 4$ cases, it concerned the tricuspid valve in intravenous drug users. Embolic events were present in $4 / 14$ cases $(28.6 \%)$, whereas in $5 / 14$ cases $(35.7 \%)$ were not mentioned.

With respect to antimicrobial treatment, in $2 / 14$ cases, it was not reported. In 7/12 (58.3\%) patients, a combination regimen based on a beta-lactam agent was started, in 5/7 cases plus aminoglycoside, in 2/7 cases plus fluoroquinolone. In the others cases, a monotherapy with a beta-lactam agent (4/12) and chloramphenicol (1/12) was prescribed.

In $3 / 14$ case, the duration of antibiotic therapy was not reported. In $7 / 11(63.6 \%)$ cases, it was equal or longer to 6 weeks while in $4 / 11(36.4 \%$ ) cases, it was inferior or equal to 4 weeks. Six out of 14 patients $(42.8 \%)$ underwent surgical intervention of valve replacement: in one case treated with monotherapy and only for 3 weeks, in 3 cases in combination therapy for 6 weeks, in 2 cases, no therapy data was available. All cases were cured, and one patient died. Eight of the 14 cases that did not undergo surgery $(57.1 \%), 6 / 8$ were cured ( $75 \%$ ) and $2 / 8$ died $(25 \%)$.

In $3 / 8(37.5 \%)$ patients who did not undergo surgical intervention and were cured, a combination therapy was administrated and its duration was equal or longer to 6 weeks.

Of all cases reported, 11/14 patients were cured $(78.6 \%)$ and $3 / 14$ died $(21.4 \%$ ) (Table 2).

\section{Discussion}

Non-HACEK (species other than Haemophilus species, Aggregatibacter actinomycetemcomitans (previously known as Actinobacillus actinomycetemcomitans), Cardiobacterium hominis, Eikenella corrodens, and Kingella species) Gramnegative bacteria (GNB) are uncommon causes of infective endocarditis. The International Collaboration on Endocarditis (ICE) study, a multinational IE registry, reported an incidence of endocarditis due to non-HACEK GNB of approximately $2 \%$ [2].

According to the AHA, in approximately half of the cases, the responsible pathogens are Escherichia coli and Pseudomonas aeruginosa. The IE due to Gram-negative non-HACEK involves a prosthetic valve in the $59 \%$ of cases. Although management included cardiac surgery in $51 \%$ of cases, the in-hospital mortality rate is $24 \%$ [3]. We did an updated review of the cases described in the literature including the review of Kalra [5-11]. After 2011, we found 6 more cases [12-17].

The mean age of study population was 54.1 years (range: 25-86 years).

As in almost all cases (71.4\%), including ours, the involved valve was the native mitral valve. 


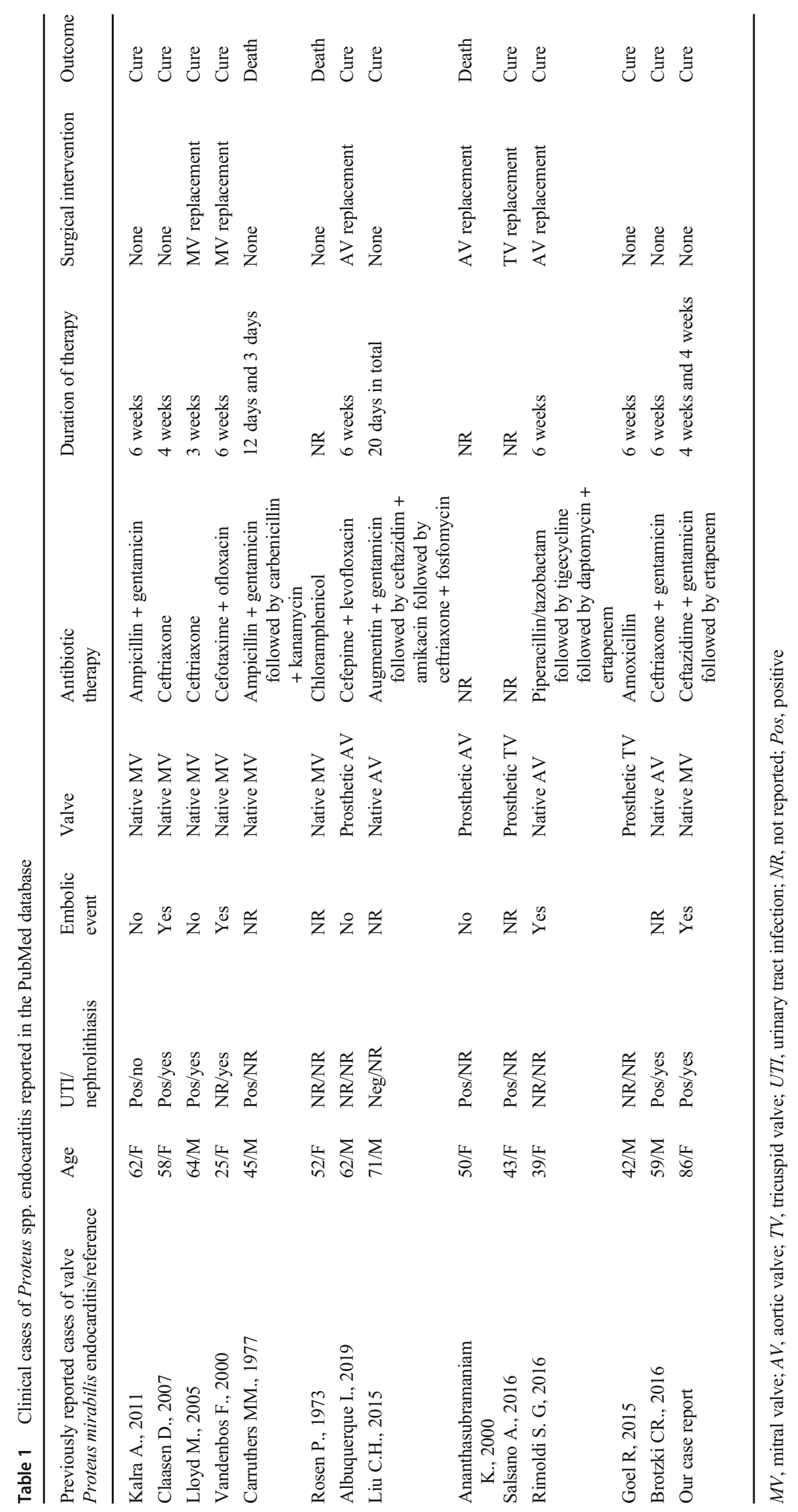


Table 2 Blood culture and sensitivity panel

\begin{tabular}{lll}
\hline Proteus mirabilis & MIC & Interpretation \\
\hline Amoxicillin/clavulanate & $\leq 2$ & $\mathrm{~S}$ \\
Amikacin & $\leq 2$ & $\mathrm{~S}$ \\
Ertapenem & $\leq 0.5$ & $\mathrm{~S}$ \\
Ciprofloxacin & 1 & $\mathrm{R}$ \\
Colistin & $>8$ & $\mathrm{R}$ \\
Cefepime & $\leq 1$ & $\mathrm{~S}$ \\
Fosfomycin & $\leq 16$ & $\mathrm{~S}$ \\
Gentamicin & $\leq 1$ & $\mathrm{~S}$ \\
Imipenem & 8 & $\mathrm{I}$ \\
Meropenem & 1 & $\mathrm{~S}$ \\
Trimethoprim-sulfamethoxazole & 160 & $\mathrm{R}$ \\
Cefotaxime & $\leq 1$ & $\mathrm{~S}$ \\
Ceftazidime & $\leq 1$ & $\mathrm{~S}$ \\
Piperacillin/tazobactam & $\leq 4$ & $\mathrm{~S}$ \\
\hline
\end{tabular}

$M I C$, minimum inhibitory concentration; $S$, sensitive; $R$, resistant; $I$, intermediate

In $88.8 \%$ cases, the patients had a concomitant urinary tract infection (UTI). Therefore, we can assert that the IE by Proteus is sustained by urinary tract infections and this could also explain the higher prevalence reported in the female population. In $83.3 \%$ cases, the patients presented nephrolithiasis which could be explained by the ability of Proteus to produce urease that convert urea to ammonia, determining an increase in $\mathrm{pH}$ value which favors the precipitation of the magnesium ammonium phosphate and calcium phosphate crystals, resulting in the formation of the renal stones [6]. In 4 cases (8.6\%), an embolic event was reported: two cases on retinal artery (6, our case), one case on the spleen [7], one case on the liver [14].

The optimal antimicrobial regimen to treat Proteus endocarditis is unknown. The scientific statement on IE from the American Heart Association (AHA) published in 2015 recommends a combination of antibiotic therapy with a beta-lactam (penicillins, cephalosporins, or carbapenem), and either a third- or fourth-generation cephalosporin or ampicillin and ciprofloxacin in patients unable to tolerate cephalosporin and ampicillin [3]. The Task Force for the Management of Infective Endocarditis of the European Society of Cardiology (ESC) recommended treatment is early surgery plus long-term (at least 6 weeks) therapy with bactericidal combinations of betalactams and aminoglycosides, sometimes with additional quinolones or cotrimoxazole [1].

Our clinical case is the sixth case among those reported in literature who was successfully treated with antimicrobial therapy alone. In our literature review, we found that in $58.3 \%$, a combination regimen was administrated according to the recommendations, while in $41.7 \%$, a monotherapy regimen was administered.
The AHA scientific statement says that cardiac surgery in combination with prolonged courses of combined antibiotic therapy ( 6 weeks) is reasonable [1]. In our review, we did not have sufficient sample sizes to assess for an association between the type of antimicrobial treatment regimen administered or whether surgery was performed and outcome.

However, we can say that all the patients who underwent surgical intervention, excluding a case in which the patient had congenital aortic insufficiency, survived and a shorter antimicrobial treatment was administered. Therefore, the $75 \%$ of patients who did not undergo surgical intervention were cured and the duration of administration was in $4 / 6$ cases equal or longer than 6 weeks. Two patients recovered with a therapy of less than 4 weeks. The 2 other cases that died were reported on 1973 and in 1977 when the antibiotic choices were limited $[9,10]$.

An important limitation of the study is that only Pubmed was searched.

\section{Conclusion}

Native endocarditis due to Proteus is rare. The major risk factor appears to be urinary tract infections with the concomitant presence of nephrolithiasis. The embolic events are not rare. The optimal antimicrobial treatment, the duration of treatment, and the association with surgery are unknown. The patients who underwent surgery have done a shorter antibiotic therapy. However, most patients were treated only with medical therapy with a good cure rate and the only two cases that died were reported in literature on 1973 and 1977. The clinical cases reported in literature are the only guide for the clinicians, but are a few sample size to assess for an association between the type of antimicrobial treatment regimen administered or whether surgery was performed and outcome; so it is more important to report the clinical case in order to provide an increase in sample size.

Authors' Contributions Conceptualization BT, SC; methodology SC; data curation BT, GP; writing - original draft preparation BT, GP, writing - review and editing BT, GP, AM, SG; visualization ES, LAM, PA, CV, MC; supervision SC; All authors have read and agreed to the published version of the manuscript.

\section{Compliance with Ethical Standards}

Conflict of Interest The authors declare that they have no conflict of interest.

Ethic Approval Not applicable.

Consent to Participate Not applicable.

Consent for Publication Not applicable. 
Open Access This article is licensed under a Creative Commons Attribution 4.0 International License, which permits use, sharing, adaptation, distribution and reproduction in any medium or format, as long as you give appropriate credit to the original author(s) and the source, provide a link to the Creative Commons licence, and indicate if changes were made. The images or other third party material in this article are included in the article's Creative Commons licence, unless indicated otherwise in a credit line to the material. If material is not included in the article's Creative Commons licence and your intended use is not permitted by statutory regulation or exceeds the permitted use, you will need to obtain permission directly from the copyright holder. To view a copy of this licence, visit http://creativecommons.org/licenses/by/4.0/.

\section{References}

1. Habib G, Lancellotti P, Antunes MJ, et al. T2015 ESC guidelines for the management of infective endocarditis the task force for the Management of Infective Endocarditis of the European Society of Cardiology (ESC). Eur Heart J. 2015;36:3075-123.

2. Murdoch DR, Corey GR, Hoen B, Miró JM, Fowler VG Jr, Bayer AS, et al. Clinical presentation, etiology, and outcome of infective endocarditis in the $21^{\text {st }}$ century: the International Collaboration on Endocarditis- Prospective Cohort study. Arch Intern Med. 2009;169:463-73.

3. Baddour LM, Wilson WR, Bayer AS, Fowler VG Jr, Tleyjeh IM, Rybak MJ, et al. Infective endocarditis in adults: diagnosis, antimicrobial therapy, and management of complications. A Scientific Statement for Healthcare Professionals From the American Heart Association. Circulation. 2015;132:1435-86.

4. Ioannou P, Vougiouklakis G. Infective endocarditis by Proteus species: a systematic review. Germs. 2020;10(4):229-39. https://doi. org/10.18683/germs.2020.1209.

5. Kalra A, Cooley C, Tsigrelis C. Treatment of endocarditis due to Proteus species: a literature review. Int J Infect Dis. 2011;4:222-5.

6. Claassen DO, Batsis JA, Orenstein R. Proteus mirabilis: a rare cause of infectious endocarditis. Scand J Infect Dis. 2007;39(4): $373-5$.
7. Vandenbos F, Hyvernat $\mathrm{H}$, Lucas $\mathrm{P}$, et al. Enterobacterial native valve endocarditis in the intensive care unit: report of two cases. Rev Med Int. 2000;6:560-1.

8. Lloyd M, Satterwhite L, Lerakis S. Successfully treated mitral valve Proteus mirabilis endocarditis. Am J Med Sci. 2005;329(5):267-9.

9. Rosen P, Armstrong D. Infective endocarditis in patients treated for malignant neoplastic diseases: a postmortem study. Am J Clin Pathol. 1973;60(2):241-50.

10. Carruthers MM. Endocarditis due to enteric bacilli other than salmonellae: case reports and literature review. Am J Med Sci. 1977;273(2):203-11.

11. Ananthasubramaniam K, Karthikeyan V. Aortic ring abscess and aortoatrial fistula complicating fulminant prosthetic valve endocarditis due to Proteus mirabilis. J Ultrasound Med. 2000;19(1):63-6.

12. Albuquerque I, Silva AR, Carreira MS, Friões F. Proteus mirabilis endocarditis. BMJ Case Rep. 2019;12:8.

13. Chun-Hao L, Wei-Jung C, Chuen C. An unusual cause of infective endocarditis: Proteus mirabilis bacteremia from an infected pressure ulcer. Int J Gerontol. 2015;9:243-5.

14. Rimoldi SG, De Vecchi E, Pagani C, et al. Use of dithiothreitol to dislodge bacteria from the biofilm on an aortic valve in the operating theatre: a case of infective endocarditis caused by Staphylococcus aureus and Proteus mirabilis. Ann Thorac Surg. 2016;102(4):357-9.

15. Brotzki CR, Mergenhagen KA, Bulman ZP, Tsuji BT, Berenson CS. Native valve Proteus mirabilis endocarditis: successful treatment of a rare entity formulated by in vitro synergy antibiotic testing. BMJ Case Rep. 2016;2016:bcr2016215956. https://doi.org/10. 1136/bcr-2016-215956.

16. Salsano A, Sportelli E, Borile S, Santini F. Proteus mirabilis bioprosthetic tricuspid valve endocarditis with massive right ventricular vegetation: a new entity in the prosthetic valve endocarditis aetiology. Eur J Cardiothorac Surg. 2016;50(3):581-2.

17. Goel R, Sekar B, Payne MN. Proteus endocarditis in an intravenous drug user. BMJ Case Rep. 2015;2015:bcr2015212447. https://doi. org/10.1136/bcr-2015-212447.

Publisher's Note Springer Nature remains neutral with regard to jurisdictional claims in published maps and institutional affiliations. 